\title{
Limpieza, corrección y geocodificación de grandes bases de direcciones utilizando minería de texto
}

\author{
Fredy Humberto Troncoso Espinosa \\ ORCID: https://orcid.org/0000-0002-9972-3123 \\ ftroncos@ubiobio.cl \\ Departamento de Ingeniería Industrial, Facultad de \\ Ingeniería, Universidad del Bío-Bío \\ Concepción, Chile
}

\author{
Nicolás Esteban Fernández Rozas \\ ORCID: https://orcid.org/0000-0002-3875-8728 \\ fernandez.rosas.nicolas@gmail \\ Investigador Independiente \\ Concepción, Chile
}

Recibido (21/04/21), Aceptado (12/05/21)

\begin{abstract}
Resumen: Para la georreferenciación de un gran número de direcciones, es necesaria la previa geocodificación mediante sistemas de carácter público o privado. La geocodificación no es una ciencia exacta porque las direcciones generalmente son escritas y almacenadas por personas, lo que provoca diferentes problemas de precisión en el registro, como errores ortográficos, datos innecesarios o falta de datos mínimos. Para enfrentar este problema, en este artículo se describe una metodología que limpia y corrige las direcciones optimizando el proceso de geocodificación utilizando los sistemas existentes. Para su desarrollo se utiliza el proceso Knowledge Discovery in Text (KDT). La metodología se aplica a una base de datos de direcciones de hechos delictivos proporcionada por la unidad de análisis penal de la Fiscalía Regional del Biobío, Chile. Los resultados muestran un aumento en el número de geocodificaciones de los sistemas implementados, que varía según el sistema utilizado.
\end{abstract}

Palabras Clave: Georreferenciación, Geocodificación, Minería de Texto.

\section{Cleaning, correcting, and geocoding of big address databases using text mining}

\begin{abstract}
For the georeferencing of a big number of addresses, prior geocoding through public or private systems is necessary. Geocoding is not an exact science because addresses are usually written and stored by people, which causes different precision issues in the registry, such as misspellings, unnecessary data, or a lack of minimal data. To address this problem, this article describes a methodology that cleans and corrects addresses by optimizing the geocoding process using existing systems. For its development, the Knowledge Discovery in Text (KDT) process is used. The methodology is applied to a database of criminal events addresses provided by the criminal analysis unit of the Regional Prosecutor's Office of Biobío, Chile. The results show an increase in the number of geocodes of the implemented systems, which varies according to the system used.
\end{abstract}

Keywords:Georreferenciación, Geocodificación, Text mining. 


\section{Introducción}

Las direcciones urbanas son el recurso más común utilizado por los habitantes de las ciudades para transmitir una posición o ubicación geográfica. Estas tienden a ser la referencia más común a eventos o fenómenos que suceden en las áreas urbanas. Con base en esto, sistemas de georreferenciación para áreas como el transporte y el tránsito, la salud pública, la seguridad pública y la recaudación de impuestos, deben generar coordenadas a partir de direcciones, para poder analizar y visualizar los datos entregados por la población [1].

La georreferenciación es un proceso que relaciona información (mapas, imágenes) con ubicaciones geográficas, mediante la utilización de las coordenadas geográficas (latitud-longitud). Para asignar una ubicación espacial a entidades cartográficas, existen dos sistemas de coordenadas el sistema de origen y el de destino. El proceso de georreferenciación está determinado por una relación de posiciones entre los elementos espaciales de ambos sistemas, de modo que, conociendo la posición de uno de los sistemas es posible conocer la posición de su sistema de coordenadas homólogo. Esta capacidad de ubicar de manera exacta las entidades geográficas es primordial para la representación cartográfica y los sistemas de información geográfica [2].

Por ejemplo, en la criminología la georreferenciación ayuda a la visualización del fenómeno, sintetizando a través de la imagen cartográfica las tendencias y lógicas de la concentración/disgregación de eventos en el espacio. En este sentido es un acierto la utilización de la georreferenciación para el monitoreo de fenómenos como robo de automóviles, casas, accidentes de tránsito, homicidios, entre otros [3].

Para georreferenciar grandes bases de datos de direcciones es necesario realizar una previa geocodificación mediante sistemas de carácter privado o público.

La geocodificación es el proceso de transformar una dirección (nombre de un lugar o alguna calle) en una posición geográfica, la cual la localiza en un punto del planeta a través de valores absolutos que no pueden ser interpretados de dos formas diferentes los cuales son la latitud y la longitud. Este proceso consta en hacer coincidir la dirección requerida con la existente en un localizador de direcciones. El localizador de direcciones es una base de datos que se utiliza para administrar información sobre la ubicación de entidades y que entrega la latitud y la longitud de estas [1].

La geocodificación no es una ciencia exacta debido a que las direcciones correspondientes al domicilio de una persona natural o jurídica son escritas por un individuo al momento de ser almacenadas en una base de datos. Esto genera diferentes problemas como faltas de ortografía, incorporación de información innecesaria o falta de información mínima [4].

Respecto a los registros de hechos delictuales, estos tienden a ser altos, por lo que la eficacia de un sistema de geocodificación es de suma importancia [5].

Este trabajo expone una metodología basada en minería de texto y elementos del procesamiento del lenguaje natural para la limpieza y corrección de direcciones, con el objetivo de complementar el trabajo de los sistemas de geocodificación y aumentar la eficiencia y eficacia del proceso de geocodificación de grandes bases de direcciones.

\section{Metodología}

A lo largo de los años, el conocimiento se ha obtenido a partir del análisis de los datos almacenados en bases de datos estructuradas. Sin embargo, la gran parte de la información está presentada en formato textual sin un orden o estructura preestablecida. El análisis y procesamiento de esta información resulta ser compleja y extensa, debido a la gran cantidad de documentos almacenados [6], su heterogeneidad y su falta de estructura [7]. Para esto se utiliza el proceso de descubrimiento de conocimiento en los textos, (KDT) [8], el cual cuenta de seis etapas las que se muestran en la figura 1.

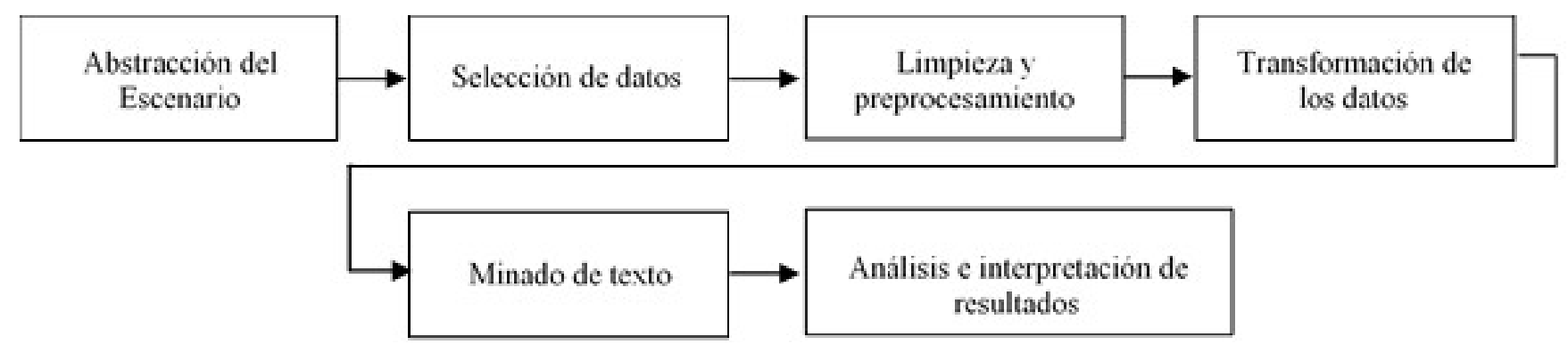

Fig. 1. Etapas metodología KDT.

La abstracción del escenario da énfasis en el entendimiento de los objetivos, requisitos, conceptos claves y problemática. La Selección de datos elige los datos disponibles para realizar el estudio e integrarlos en uno solo y así mejorar la capacidad de análisis de los objetivos. La limpieza y preprocesamiento da homogeneidad al texto, se busca que no existan conceptos o caracteres que distorsionen posteriormente la detección de patrones. La transformación de los datos da la estructura necesaria al texto para la posterior detección de patrones o minería de texto, algunos procesos que se pueden aplicar son el lematizado (trasformar las variaciones de las palabras con morfemas a su raíz con el fin de evitar tener variantes de una misma palabra) el etiquetado (que es el proceso de clasificar las palabras en función tanto de su definición 
como de su contexto) y tokenizado (que es el proceso de partir el texto en elementos indivisibles llamados tokens).

El minado de texto permite el descubrimiento del conocimiento, el cual se puede dar a través de la detección de patrones, representaciones vectoriales, modelos de aprendizaje supervisado o no supervisado entre otros métodos. El análisis e interpretación de resultados interpreta y valida el conocimiento obtenido tras realizar el proceso de minería de texto. En el caso de que el analista no valide el conocimiento, es necesario retroceder a las etapas anteriores para realizar un cambio que genere un nuevo resultado.

Sobre la base de este proceso, se propone una metodología de limpieza de direcciones la cual se explica en el siguien- te apartado.

\section{Desarrollo}

Las direcciones suelen ser textos bastantes cortos, sin embargo, es necesario buscar un patrón que permita identificar los principales errores en la escritura de las direcciones de manera de que estos sean corregidos automáticamente y mejorar la eficacia de los sistemas de geocodificación. Por esto, se propone una metodología de ocho etapas basada en el proceso de descubrimiento de conocimiento del texto (KDT). La figura 2 muestra las etapas de la metodología creada.

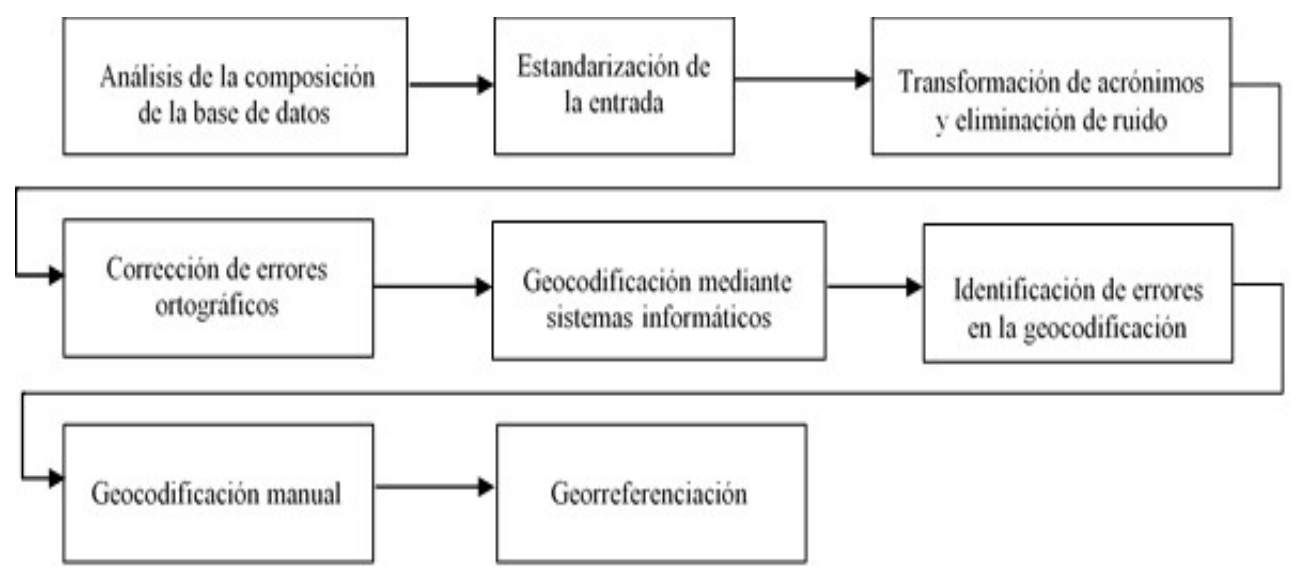

Fig. 2. Etapas de la metodología de limpieza de direcciones.

A.Análisis de la composición de la base de datos: Esta etapa consta de determinar la composición de la base de datos identificando los atributos que está posee y si contiene atributos necesarios para realizar una correcta geocodificación, en caso de no contar con los atributos mínimos necesarios se deben recopilar los datos faltantes, sin estos no se puede realizar una geocodificación correcta.

B.Estandarización de la entrada: Se debe realizar un proceso de estandarización de la entrada, debido a que la información se puede presentar de diferentes formas. Esta puede presentarse en una sola cadena de texto que contiene la dirección, número de domicilio y comuna de residencia, todos estos atributos por separado o una combinación de estos.

C.Transcripción de acrónimos y eliminación de ruido: Los acrónimos en las direcciones son recurrentes. Palabras como "avenida", "calle", "parcela", "pasaje", etc. son frecuentemente escritos por sus acrónimos. Debido a que no existe un solo acrónimo por palabra, es necesario reemplazar los acrónimos por sus palabras originales de manera de facilitar la corrección de errores ortográficos y realizar un análisis de patrones mucho más efectivos. Para el proceso de eliminación del ruido es necesario eliminar registros, caracteres o texto que no añaden valor para la geocodificación.

D.Corrección de errores: la corrección de errores ortográficos es necesaria, ya que estos producen errores en la geocodificación y disminuyen la capacidad del sistema. Este proceso se realiza cruzando la base de datos que se desea geocodificar con un maestro de calles, el cual es una base de datos que contiene el nombre de las calles según la ley.

E.Geocodificación: Este proceso se realiza la geocodificación de direcciones mediante algún sistema de geocodificación de carácter privado o público.

F.Identificación de errores en la geocodificación: la geocodificación no es una ciencia exacta debido a que las direcciones no siempre se encuentran en el localizador de direcciones de los sistemas de geocodificación. Por ende, se generan errores de geocodificación, geocodificando direcciones en lugares erróneos. Por esto es necesario realizar un proceso de identificación y extracción de direcciones mal geocodificadas.

G.Geocodificación manual: Las direcciones que no han podido ser geocodificadas o que han sido geocodificads en 
forma incorrecta deben pasar por un proceso de revisión de la calidad de la dirección para ver si esta puede ser corregida, luego ingresarla al geocodificador o geocodificarlas manualmente.

H.Georreferenciación: Este proceso determina la posición de un punto en un sistema de coordenadas espacial mediante la utilización de las coordenadas geográficas latitud-longitud. Este proceso permite realizar un análisis visual e identificar si existen puntos erróneos.

\section{Resultados}

A continuación, se presentan los resultados de la metodología propuesta, aplicada a una base de datos de ubicaciones de eventos criminales proporcionada por la Unidad de Análisis Criminal y Focos Investigativos de la Fiscalía Regional del Biobío, de la ciudad de Concepción, Chile. Para la implementación metodológica se utilizó el lenguaje de programación Python 3.0 [9] [10].

\section{A.Análisis de la composición de la base de datos}

La fiscalía de concepción se encarga de las necesidades de la Región del Bio-Bío en materia de investigación penal, almacena todos los datos correspondientes a las direcciones en donde ha ocurrido un suceso delictual. La georreferenciación de los hechos criminales es necesaria para la busque da de parones criminales y de puntos calientes en los cuales concentran la actividad criminal.

La base de datos de la fiscalía está construida con 15337 direcciones de sucesos delictuales ocurridos en las Regiónes del Bio-Bío y Nuble en el año 2016. Esta base de datos cuenta con los atributos "nombre de la calle", "número del domicilio", "nombre de la comuna", tipo de delito.

Al revisar la base de datos se encontró anomalías como errores de digitación, formato, abreviaciones, faltas de ortografía y acrónimos. Estas anomalías generan errores al utilizar sistemas de geocodificación.

\section{B.Estandarización de la entrada}

Los datos pueden ser presentados de diferentes maneras, dada la no existencia de un estándar en la forma de registrar las direcciones. La tabla I muestra el formato estándar utilizado para el registro de las direcciones en esta investigación.

Tabla 1. Formato de Estandarizado.

\section{Nombre de la calle Número del Domicilio Comuna}

Avenida los Carrera $\quad 30102$

Concepción

\section{C.Transformación de acrónimos y eliminación de ruido}

Para la corrección de acrónimos se realizó un análisis visual de la composición de las direcciones y sus acrónimos más frecuentes. A partir de este análisis se distinguieron los acrónimos más frecuentes para su corrección.

Para operar con textos es necesario que las cadenas de texto tengan el mismo formato. Por esta razón se transformó todo el texto a mayúscula, se eliminó tildes y espacios innecesarios.

Se realizó la limpieza de símbolos y contenido que no aporta valor a la dirección como intersecciones de calles o comentarios. También se eliminó todas aquellas direcciones que no correspondían a la zona a geocodificar.

\section{Corrección de errores ortográficos}

Para corregir los errores ortográficos se cruzaron las direcciones de la base de datos con las direcciones escritas correctamente. Para esto e confeccionó un maestro de calles, es decir, una base de datos con las direcciones de la zona geográfica correctamente escritas. Para su construcción se consideraron tres fuentes de información: el maestro de calles año 2018 del Instituto Nacional de Estadísticas de Chile (INE), la plataforma codigopostal.org y OpenAddresses.
Con el fin de realizar las correcciones de los errores, se recurrió a técnicas que miden la distancia de edición entre dos cadenas de texto. La distancia de edición entre dos cadenas de texto A y B es el mínimo costo s de una secuencia de pasos de edición, dentro de las que se encuentra inserciones, eliminaciones, sustitución y cambios que convierten una cadena en otra [11].

Para medir esta distancia fue utilizado el algoritmo de Levenshtein el cual es un algoritmo de programación dinámica [12] [13].

Se determinó el valor máximo de operaciones aceptables sin que la aplicación del algoritmo de Levenshtein generara errores en la corrección ortográfica de las direcciones. Para determinar este valor se seleccionó una muestra aleatoria de 2000 direcciones y en forma manual se analizó el número de errores generados. La tabla II muestra que el número óptimo de cambios es una sola edición con un acierto del 99\%. 
Tabla 2. Número de cambios Levenshtein.

\begin{tabular}{ccccc}
\hline N' Cambios & Correctos & Incorrectos & Total & $\begin{array}{c}\text { Porcentaje } \\
\text { de } \\
\text { Correctos }\end{array}$ \\
\hline 1 & 117 & 1 & 118 & $99 \%$ \\
2 & 28 & 17 & 45 & $62 \%$ \\
3 & 20 & 63 & 83 & $24 \%$ \\
4 & 12 & 79 & 91 & $13 \%$ \\
\hline
\end{tabular}

\section{E.Geocodificación}

Existen diferentes sistemas de geocodificación. En la tabla III se muestran algunos sistemas de geocodificación disponibles en el mercado [14] [15] [16] [17] [18] [19] [20].
Para probar la metodología propuesta, se consideró los geocodificadores Bing Maps y Open Street Map.

Tabla 3. Algunos geocodificadores del mercado.

\begin{tabular}{cccc}
\hline $\begin{array}{c}\text { Siste mas de } \\
\text { Geocodificación }\end{array}$ & $\begin{array}{c}\text { Requiere } \\
\text { programació } \\
\text { n }\end{array}$ & $\begin{array}{c}\text { Número de } \\
\text { geocodificaciones } \\
\text { gratuitas }\end{array}$ & $\begin{array}{c}\text { Calidad de la } \\
\text { información }\end{array}$ \\
\hline Bing Maps & si & $\begin{array}{c}50.000 \text { diarias y } 150.000 \\
\text { anuales }\end{array}$ & Alta \\
Open Street Map & si & Ilimitadas & $\begin{array}{c}\text { Media Alta (depende } \\
\text { de la zona) }\end{array}$ \\
Yahoo PlaceFinder & si & ilimitadas de pago & Alta \\
Opencage & si & 2500 diarias & Media \\
Mapquest & si & 15,000 por mes & Media Alta (depende \\
de la zona) \\
Google My Maps & no & 2000 diarias & Alta \\
Google Geocoding API & si & ilimitadas de pago & Alta \\
OpenAddreses & si & ilimitadas & Media Baja \\
\hline
\end{tabular}

F.Identificación de errores en la geocodificación

La geocodificación se complementó con un sistema para la identificación y contabilización de errores de elaboración propia, de manera de facilita el análisis de los resultados. Este sistema identifica una dirección geocodificada erróneamente al comparar los campos país, región y comuna.
Para evaluar el desempeño de la metodología propuesta, se evaluó el número de geocodificaciones realizadas y el número de geocodificaciones correctas realizadas utilizando y no utilizando la metodología. La tabla IV muestra los resultados de la geocodificación para ambos casos

Tabla 4. Resultados geocodificación sin aplicar la metodología de limpieza propuesta.

\begin{tabular}{ccccc}
\hline & \multicolumn{2}{c}{ Sin la metodología propuesta } & \multicolumn{2}{c}{$\begin{array}{c}\text { Con la metodología } \\
\text { propuesta }\end{array}$} \\
\cline { 2 - 5 } & Open Street Maps & Bing Maps & Open Street Maps & Bing Maps \\
\hline $\begin{array}{c}\text { Número de direcciones } \\
\text { Geocodificadas }\end{array}$ & 12520 & 15337 & 12489 & 15311 \\
$\begin{array}{c}\text { Número de direcciones no } \\
\text { Geocodificadas }\end{array}$ & 2817 & 0 & 2824 & 2 \\
Geocodificados Incorrectos & 1234 & 3219 & 205 & 1698 \\
Geocodificados Correctos & 11286 & 12118 & 12284 & 13613 \\
\hline
\end{tabular}


Se observó que sin utilizar la metodología propuesta Open Street Maps y Bing Maps geocodificaron correctamente 11286 y 12118 direcciones respectivamente de las 15337 consideradas. Lo que corresponde a un $73.58 \%$ con Open Street Maps y a un 79\% utilizando Bing Maps.

Utilizando la metodología propuesta, Open Street Maps y Bing Maps geocodificaron correctamente 12284 y 13613 direcciones respectivamente, de las 15337 consideradas. Lo que corresponde a un $88.76 \%$ utilizando Bing Maps y a un $80.09 \%$ utilizando Open Street Maps. El aumento en la cantidad de geocodificaciones correctas entregadas por Open Street Maps fue de un 8.84\% y el aumento en las geocodificaciones correctas entregadas por Bing Maps fue de un 12.33\%.

\section{G.Geocodificación manual}

La geocodificación manual consta de trascribir la dirección en Google Maps o alguna otra plataforma y guardar la latitud y longitud en una base de datos. Se estima el tiempo necesario para este procedimiento en por lo menos 30 segundos por dirección. Las direcciones que no pudieron ser geo- codificadas o que fueron geocodificadas en forma incorrecta no contenían información suficiente para ser geocodificadas manualmente, por lo que fueron descartadas.

\section{H.Georreferenciación}

Para el proceso de georreferenciación de la base de datos, se utilizó información entregada por la Universidad de la Frontera de Temuco, Chile en su plataforma online, la cual es un mapa correspondiente a la actual región del Bio-Bío y la región de Nuble, que contiene las demarcaciones por comuna.

El sistema empleado para la georreferenciación en el entorno Python fue Geopandas [21] el cual es un proyecto open source. La georreferenciación tubo un tiempo de ejecución de tres minutos para la totalidad de la base de datos.

En la figura 3 se aprecia con puntos rojos las direcciones que fueron georreferenciadas de la base de datos de la fiscalía. La totalidad de direcciones geocodificadas fue georreferenciada dentro del área regional.

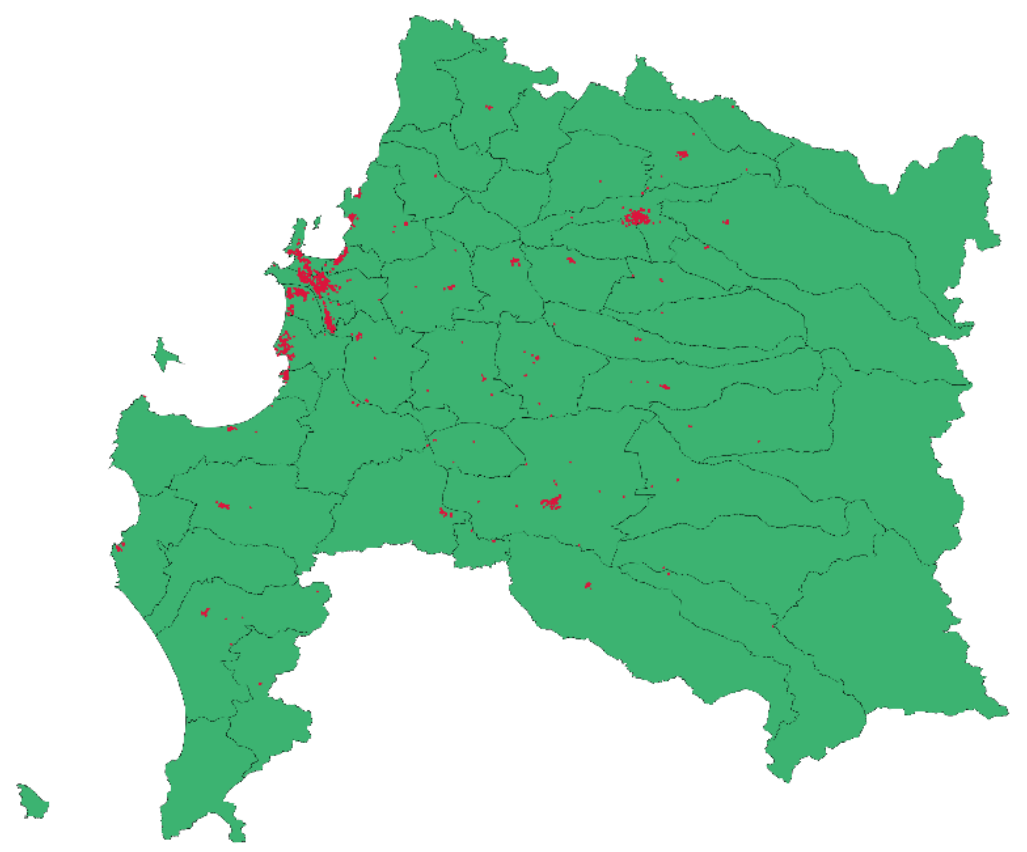

Fig.3. Georreferenciación de las direcciones procesadas Regiones del Bío-Bío y Ñuble, Chile.

\section{I.Tiempos de ejecución}

El tiempo empleado en la georreferenciación se ve reducido en gran medida con el uso de sistemas de geocodificación. La tabla V muestra los tiempos de ejecución de los sistemas utilizados complementados con la metodología propuesta y la reducción de tiempo de cada geocodificador respecto a la geocodificación manual. Los tiempos ejecución de los sistemas de geocodificación muestran una reducción considerable respecto a la geocodificación manual. 
Tabla 5. Tiempo de ejecución de los tipos de geocodificación.

\begin{tabular}{ccccccc}
\hline $\begin{array}{c}\text { Geocodificad } \\
\text { or }\end{array}$ & $\begin{array}{c}\text { Tiempo de } \\
\text { ge ocodificació } \\
\text { n (horas) }\end{array}$ & $\begin{array}{c}\text { Limpieza } \\
\text { de } \\
\text { direccione } \\
\text { s (horas) }\end{array}$ & $\begin{array}{c}\text { Tiemp } \\
\text { o total } \\
\text { (horas } \\
\text { ) }\end{array}$ & $\begin{array}{c}\text { Tiempo } \\
\text { por } \\
\text { dirección } \\
\text { (segundo } \\
\text { s) }\end{array}$ & $\begin{array}{c}\text { Tiempo } \\
\text { reducid } \\
\text { o }\end{array}$ & $\begin{array}{c}\text { Reducción } \\
\text { de tiempo } \\
\text { (\%oras) }\end{array}$ \\
\hline Manual & 127,80 & No & & & & \\
Open Street Maps & 4,2 & determinado & 127,80 & 30 & - & - \\
Bing Maps & 2,5 & 2,3 & 6,5 & 1,5 & 121,30 & $94,9 \%$ \\
\hline
\end{tabular}

\section{J.Discusión}

La aplicación de la metodología de limpieza y corrección de direcciones generó un incremento en el número de geocodificaciones entregados por los sistemas de geocodificación utilizados. En el caso de Open Street Maps hubo una mejora de un $8.8 \%$ y en el caso de Bing Maps de un 12.3\%.

Al implementar ambos sistemas de geocodificación, se observó que el sistema Bing Maps es superior en tiempos de ejecución y capacidad de geocodificación que el sistema de Open Street Maps.

En cuanto a los tiempos de ejecución de los sistemas de geocodificación complementados con la metodología propuesta, se observa una mejora considerable, no inferior al $94 \%$, independiente del sistema utilizado. Esto se debe a que la geocodificación manual es tardía y poco eficiente.

Al implementar la metodología propuesta, se redujo la necesidad de georreferenciar manualmente las direcciones en un $25,22 \%$ en Open Street Maps y un 47,18\% en el caso de Bing Map. Esto implica una reducción significativa de los recursos involucrados en la tarea de georreferenciación.

En el proceso de georreferenciación, se logra visualizar que las direcciones, después aplicar la metodología fueron geocodificadas de una manera correcta, debido a que ninguna se visualiza fuera del perímetro de la región del Bio-Bío y la región del Ñuble.

\section{Conclusiones}

La metodología propuesta se basa en técnicas de limpieza, transformación, procesamiento del lenguaje natural y minería de texto. Su aplicación permite mejorar los resultados obtenidos por los sistemas de geocodificación mediante la aplicación complementaria de cada una de sus ocho etapas, las cuales agregan valor al proceso.

La aplicación de esta metodología muestra una mejora promedio en la capacidad geocodificación de un $11 \%$, lo que depende del sistema de geocodificación utilizado.

La metodología propuesta es una herramienta eficiente que permite maximizar la cantidad de geocodificaciones y reducir el tiempo del proceso de geocodificación considerando los errores, independiente del área a la cual sea aplicada.

La mejora en el sistema de corrección de direcciones depende en gran medida del maestro de calles. Contar con un maestro de calles más completo permitiría mejorar el número de direcciones geocodificadas.
Identificar cuál es el mejor sistema de geocodificación para la localidad que se desea geocodificar es primordial. Existen sistemas de sistemas de geocodificación que no cuentan con todas las direcciones de todas las localidades dentro de su base de datos.

\section{Reconocimiento}

Se agradece el apoyo financiero otorgado por la Universidad del Bío-Bío, Chile, mediante el Proyecto de Iniciación en Investigación código 2060204IF/I y a la Macrofacultad de Ingeniería 2030, Chile, mediante el Proyecto código ING 2030 I+D 20-34. Se agradece también el apoyo técnico y los datos facilitados por la Unidad de Análisis Criminal de la Fiscalía Regional del Biobío, Chile mediante un acuerdo de colaboración.

\section{Referencias}

[1]C. Davis y F. Fonseca, «Assessing the Certainty of Locations Produced by an Address Geocoding System,» Geoinformatica, vol. 11, pp. 103-129, 2007.

[2]L. Hill, «Georeferencing in Digital Libraries,» D-Lib Magazine, vol. 10, $\mathrm{n}^{\circ}$ 5, 2004.

[3]J. Pontón y A. Santillán, «Seguridad Ciudadana: escenarios y efectos,» 2008.

[4]D. W. Goldberg, «Spatial approaches to reducing error in geocoded data,» 2010.

[5]D.-H. Yang, L. M. Bilaver, O. Hayes y R. Goerge, «Improving Geocoding Practices: Evaluation of Geocoding Tools,» Journal of Medical Systems, vol. 28, pp. 361-370, 2004.

[6]T. Ah-Hwee, «Text mining: The state of the art and the challenges,» de PAKDD'99 workshop on Knowledge Discovery from Advanced Databases, Beijing, 1999.

[7]R. Feldman y I. Dagan, «Knowledge discovery in textual databases,» de First International Conference on Knowledge Discovery and Data Mining (KDD-95), 1995.

[8]M. d. C Justicia de la Torre, «Nuevas Tecnicas de Mineria de Textos: Aplicaciones,» Granada, 2017.

[9]M. Lutz, Programming Python, vol. 2, O'reilly \& Associates, 2001, pp. 1-10.

[10]W. McKinney, Python For Dara Analysis, O'Reilly, 2012, pp. 111-152.

[11]E. Ukkonen, «Algorithms for Approximate String Matching,» de International Conference on Foundations of Com- 
putation Theory, 1985.

[12]M. A. Alvarez Carmona, «Deteccion de similitud en textos cortos considerando traslape, ordeny relacion semantica de palabras,» Tonantzintla, Puebla, 2014.

[13]V. I. Levenshtein, «Binary Codes Capble Of Correcting Deletions, Insertions, and Reversals,» Soviet Physics Doklady, vol. 10, p. 707, 2 February 1966.

[14]Google, «Google Maps Plataform,» 2020. [En línea]. Available: https://developers.google.com/maps/documentation/javascript/geocoding?hl=es-419. [Último acceso: 29 Julio 2020].

[15]Mapquest, «Mapquest Developer,» 2020. [En línea]. Available: https://developer.mapquest.com/. [Último acceso: 25 Julio 2020].

[16]Microsoft Corporation, «Bing Maps Dev Center,» 2020.
[En línea]. Available: https://www.bingmapsportal.com/. [Último acceso: 29 Julio 2020].

[17]Open Street Map Wiki, 2020. [En línea]. Available: https://wiki.openstreetmap.org/wiki/Main_Page. [Último acceso: 29 Julio 2020].

[18]OpenAdrdresses, «OpenAdrdresses,» 2020. [En línea]. Available: https://openaddresses.io/. [Último acceso: 25 Julio 2020].

[19]OpenCage Geocoder, 2020. [En línea]. Available: https:// opencagedata.com/. [Último acceso: 29 Julio 2020].

[20]Yahoo, «Yahoo Developer,» 2016. [En línea]. Available: https://developer.yahoo.com/. [Último acceso: 14 Agosto 2020].

[21]K. Jordahl, J. Van Den Bossche y J. Wasserman, «Geopandas/Geopandas: V0. 4.1. Zenodo,» 2020.

\section{RESUMEN CURRICULAR}

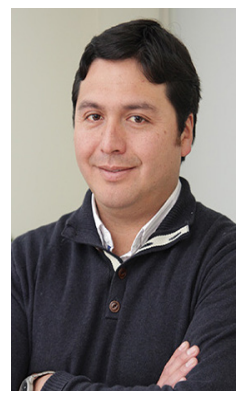

Fredy Troncoso Espinosa, Doctor en Sistemas de Ingeniería, Universidad de Chile, Ingeniero Civil Industrial Universidad del Bío-Bío, Chile. Académico e Investigador Departamento de Ingeniería Industrial, Universidad del Bío-Bío. Concepción, Chile

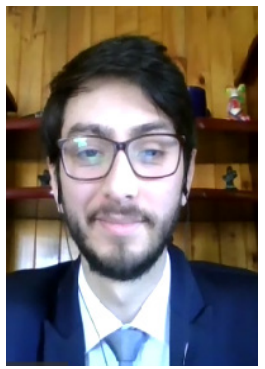

Nicolás Fernández Rozas, Ingeniero Civil Industrial, Universidad del Bío-Bío, Investigador Independiente. Chile 